\title{
Coarse-Grained Molecular Dynamics: Dissipation Due to Internal Modes
}

Robert E. Rudd

This article was presented to: 2001 Materials Research Society Fall Meeting, Boston MA, November 26-30, 2001

\section{December 21, 2001}




\section{DISCLAIMER}

This document was prepared as an account of work sponsored by an agency of the United States Government. Neither the United States Government nor the University of California nor any of their employees, makes any warranty, express or implied, or assumes any legal liability or responsibility for the accuracy, completeness, or usefulness of any information, apparatus, product, or process disclosed, or represents that its use would not infringe privately owned rights. Reference herein to any specific commercial product, process, or service by trade name, trademark, manufacturer, or otherwise, does not necessarily constitute or imply its endorsement, recommendation, or favoring by the United States Government or the University of California. The views and opinions of authors expressed herein do not necessarily state or reflect those of the United States Government or the University of California, and shall not be used for advertising or product endorsement purposes.

This is a preprint of a paper intended for publication in a journal or proceedings. Since changes may be made before publication, this preprint is made available with the understanding that it will not be cited or reproduced without the permission of the author.

This report has been reproduced

directly from the best available copy.

A vailable to DOE and DOE contractors from the

Office of Scientific and Technical Information

P.O. Box 62, Oak Ridge, TN 37831

Prices available from (423) 576-8401

http:/ / apollo.osti.gov/ bridge/

A vailable to the public from the

National Technical Information Service

U.S. Department of Commerce

5285 Port Royal Rd.,

Springfield, VA 22161

http:/ / www.ntis.gov/

OR

Lawrence Livermore National Laboratory

Technical Information Department's Digital Library

http:/ / www.Ilnl.gov/ tid/ Library.html 


\title{
Coarse-Grained Molecular Dynamics: Dissipation due to Internal Modes
}

\author{
Robert E. Rudd \\ Lawrence Livermore National Laboratory \\ Condensed Matter Physics Division, L-045 \\ Livermore, CA 94551 USA
}

\begin{abstract}
We describe progress on the issue of pathological elastic wave reflection in atomistic and multiscale simulation. First we briefly review Coarse-Grained Molecular Dynamics (CGMD). Originally CGMD was formulated as a Hamiltonian system in which energy is conserved. This formulation is useful for many applications, but recently CGMD has been extended to include generalized Langevin forces. Here we describe how Langevin dynamics arise naturally in CGMD, and we examine the implication for elastic wave scattering.
\end{abstract}

\section{INTRODUCTION}

Multiple scales arise in many physical systems. Much of the richness of materials science is due to the endless combinations of interplay between processes at diverse scales, including cooperative dynamics and competition. The multiscale modeling tools developed in recent years have proven to be an effective way to treat the length scale problem. In most cases studied to date, multiple length scales are treated sequentially: ab initio calculations are used to develop classical interatomic potentials that are in turn used in large-scale molecular dynamics (MD) simulations that form the basis for mesoscale and continuum models such as finite element (FE) models. This approach works well when the scales are weakly coupled. When the scales are strongly coupled, multiple scales must be treated simultaneously. This has become known as concurrent multiscale modeling. [1]

Coarse-grained molecular dynamics (CGMD) is an example of concurrent multiscale modeling that is optimized to simulate dynamical and finite temperature processes at, and above, the nanoscale. It unifies atomistic simulation with a generalization of finite element modeling in a concurrent simulation. [2] The methodology is designed for inhomogeneous systems with regions that require the precision of a full, atomistic description and regions that are well modeled by a simplified description. An example of such a system is the microresonator shown in Fig. 1. [3] The resonator is a bar of semiconductor that has been etched from a single crystal. The etching has released the bar from the substrate so that it is free to oscillate much like a violin string. Bridge-type resonators of this general design have been used in a variety of Micro-Electro-Mechanical Systems (MEMS). [4] Currently several experimental groups are developing sub-micron resonators for use in Nano-Electro-Mechanical Systems (NEMS). [5,6] The NEMS resonator is an archetypical system with two relevant length scales. The width of the bar can be less than 100 atoms, so nanoscale atomistic effects can be important; at the same time, elastic fields extend from the resonating bar for microns out into the substrate. These elastic fields are well described by continuum mechanics. [3] CGMD allows both the atomistic and the continuum physics to be modeled concurrently in a single simulation. 

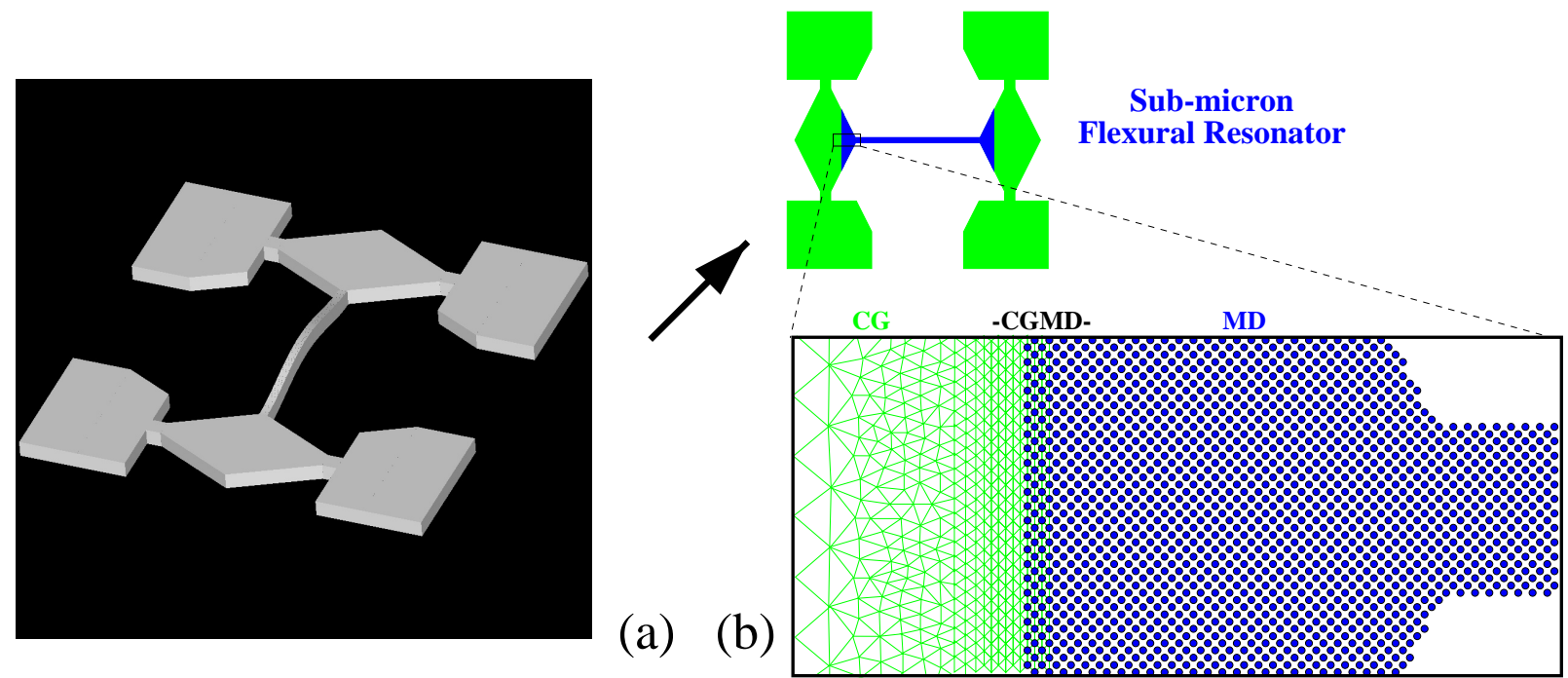

Figure 1: (a) Three dimensional model of the microresonator in its fully deflected state. The geometry is modeled on a device fabricated by Roukes and coworkers [5]. (b) The decomposition of the microresonator system into molecular dynamics (MD) and coarsegrained (CG) regions. The cross-over to atomistics for atomic-sized cells is completely smooth and no handshaking region is required. [1,2]

\section{COMPUTATIONAL METHODOLOGY}

The basic principle of CGMD is to use, in each spatial region of such a heterogeneous system, a description that is as simple as possible, but of course no simpler. In practice this is implemented through the introduction of a mesh reminiscent of finite element meshes. The size of the mesh determines the physics that is modeled. In some regions, the nodes of the mesh are in one-to-one correspondence with the atomic sites, taken to be the equilibrium positions of the atoms in the undeformed material. We will refer to these as the MD regions. In other regions, the mesh spacing is larger so that some of the atomic-level detail is eliminated with a commensurate gain in computational efficiency. These are the coarse-grained (CG) regions. This much of the formalism is identical to hybrid atomistic/continuum (MD/FE) techniques that employ conventional finite element models coupled to MD [1,7], and it is similar to the approach taken in the Quasi-Continuum Technique. [8] The distinction with CGMD is that the model used in the $\mathrm{CG}$ region is derived from the dynamics of the underlying atomistic model. Short wavelength modes are integrated out of the model of the coarse-grained region to produce an effective model of the relevant long wavelength modes. This ensures that the dynamic coupling between the MD and CG regions is as seamless as possible. [2]

It was shown in Ref. 7 that the coupling of MD with conventional finite elements is practically seamless for some important applications. In particular, shock waves emitted from a crack in the MD region of a thin slice of silicon were shown to propagate out 
through the interface to the FE region without significant back-scatter: absent was the appreciable effect of reflected waves on the crack tip velocity encountered in simulations with periodic or fixed boundary conditions [9]. Of course, this required the $\mathrm{MD} / \mathrm{FE}$ interface to be sufficiently far removed from the crack tip. In order to bring the interface closer and, hence, to reduce further the number of simulated degrees of freedom, it is necessary to improve the fidelity of the interface. In a perfect coupling, the atomistic forces would be recovered smoothly as the size of a finite element cell is reduced to the atomic scale. This is not achieved in practice with conventional finite element models because the continuum mechanics does not have any information about the crystallography.

The absence of spurious elastic wave reflections is just one of the properties that is demanded of CGMD. It also meets three other general requirements: elastic, thermal and dissipative properties must be physical in the CG region. In particular, CGMD guarantees that strain fields, local temperature and relaxation rates vary smoothly across the interface where the mesh is coarsened. [2]

\section{The Model}

We turn now to the details of the model, both without and with random, dissipative forces. The Hamiltonian formulation of CGMD is derived from the underlying atomistic model using statistical mechanical techniques to integrate out the short wavelength degrees of freedom. For the purposes of this discussion a short wavelength mode is a wave whose wavelength is less than the local mesh spacing. Such a wave cannot be represented on that region of the mesh. Typically, a mesh will have both forbidden and allowed regions for waves of a particular wavelength. The averaging procedure that integrates out the short wavelength modes uses perturbation theory about a harmonic lattice. In the zeroth order (harmonic approximation), the CGMD equations are

$$
\begin{aligned}
\vec{u}_{i} & =G_{i j} \vec{f}_{j} \quad \text { (static) } \\
M_{i j} \ddot{\vec{u}}_{i} & =-G_{i k}^{-1} \vec{u}_{k} \quad \text { (dynamic) }
\end{aligned}
$$

where $\vec{u}_{i}$ and $\vec{f}_{i}$ are the CG displacement field and body force at node $i$, and $M_{i j}$ and $G_{i j}$ are the CG mass matrix and elastic Green's function, respectively. In higher orders of perturbation theory, nonlinear terms appear in the force law (2). [1] For a monatomic lattice with atomic mass $m_{\mu}$ for atom $\mu$ and lattice Green's function $G_{\mu \nu}$, the mass matrix and elastic Green's function are given by

$$
\begin{aligned}
M_{i j} & =N_{i \mu} m_{\mu} N_{j \mu} \\
G_{i j} & =g_{i k}^{-1} N_{k \mu} G_{\mu \nu} N_{l \nu} g_{j l}^{-1}
\end{aligned}
$$

where $N_{i \mu}=N_{i}\left(x_{0 \mu}\right)$ is the CGMD shape function, $g_{i j}=N_{i \mu} N_{j \mu}$ is the CG metric, and repeated indices are summed. Apart from factors required for covariance, Eqs. (3) and (4) state that the CGMD mass and compliance are just weighted averages of the underlying atomistic masses and compliances, where the weighting function is the CGMD shape function, $N_{i \mu}$. See Refs. 2 and 1 for details.

The model as it has been described thus far is based on an effective Hamiltonian, and it conserves energy. It has a number of nice properties as a coarse-grained model. The 
phonon energy spectrum is a very good approximation to the long wavelength part of the spectrum in the underlying atomistic model. [2] In some simple cases the spectrum may be computed exactly. In the case of an infinite chain of harmonic atoms coarse-grained with linear interpolation to a commensurate lattice (i.e. a fixed number $\nu$ of atoms per node), the frequency, $\omega$, as a function of wave number, $k$, is given by [2]

$$
\omega(k)=2 \sqrt{\frac{K}{m}}\left(\frac{\sum_{p=0}^{\nu-1} \sin ^{-4}\left(\frac{1}{2} k a+\pi p \nu\right)}{\sum_{p=0}^{\nu-1} \sin ^{-6}\left(\frac{1}{2} k a+\pi p \nu\right)}\right)^{1 / 2}
$$

where $K$ is the force constant. This spectrum is improved over the spectrum in conventional finite element analysis in both the long and short wavelength regime. It may be of some interest to note that the form of the spectrum is familiar as a character corresponding to the broken discrete translation group.

Dissipation. If a wave propagates from a region where it is represented into a region where it is not, it will be rejected. In the energy-conserving formulation described above, the wave is reflected. The change in the mesh spacing breaks translation invariance, so momentum is not conserved. Energy is, however, so the wave must go somewhere, and reflection results. A closely related problem is the fact that no short wavelength modes can be emitted from a coarse region of the mesh. The short wavelength part of the spectrum is not populated by a random thermal distribution, but only by reflected waves. These waves are highly correlated with the wave incident on the coarse region, and they may be far from equilibrium. The reflection and spectrum distortion are artifacts of the coarse-graining procedure, and they are indications that the formulation is incomplete. These effects may be benign, but in cases where the CG/MD interface is very close to the region of interest (such as the tip of a crack), they can cause pathologies in the quantities of interest.

The missing ingredients are random and dissipative forces. [2,1] These forces are well known from Brownian motion, where a heavy particle experiences drag forces and random buffeting due to the surrounding bath of relatively light molecules. In CGMD, too, the relatively heavy CG modes interact with the internal modes that have been integrated out. The resulting equation of motion is a generalized Langevin equation. The random and dissipative forces solve deficiencies of the conservative model for outgoing and incoming waves. Firstly, short wavelength modes incident on a part of the mesh where they are not supported are not reflected but absorbed. Secondly, incoming elastic waves are generated randomly at the interface to fill out the thermal spectrum. Finally, short wavelength modes that would propagate through the CG region and reappear at an MD region on the far side (for example due to periodic boundary conditions), may or may not do so depending on whether the memory is implemented with propagators or not. CGMD offers this choice, and each option may be advantageous depending on the application.

The equations of motion for CGMD with random and dissipative forces are derived using projection operator techniques. [10] The details are beyond the scope of this article, and will be presented elsewhere. [11] The result is a generalized Langevin equation

$$
M_{i j} \ddot{\vec{u}}_{j}=-G_{i k}^{-1} \vec{u}_{k}+\int_{-\infty}^{t} d t^{\prime} \eta_{i k}\left(t-t^{\prime}\right) \dot{\vec{u}}_{k}\left(t^{\prime}\right)+\vec{F}_{i}(t) .
$$

where $\eta_{i k}(\Delta t)$ is a memory function and $\vec{F}_{i}$ is a force that is taken to be random. We need to determine $\eta(t)$ and $F(t)$. If the forces in the CG region are approximately harmonic, 
then it is possible to derive an expression for $\eta_{i k}$ in terms of the inverse Laplace transform of a certain spectral function. In practice, we have used a lumped mass approximation for $M_{i j}$ to allow explicit integration of the equations of motion. $[1,12]$

\section{RESULTS AND VALIDATION}

For the purposes of this article, we focus on new results of the validation of the non-conservative formulation of CGMD. The immediate goal of the Langevin CGMD is to eliminate unphysical elastic wave scattering off the CG interface. In order to quantify this effect, we have calculated the reflection coefficient for waves scattering from a coarse-grained region in the middle of an infinite chain of atoms. The largest cell in the CG region consists of 20 atoms. The same configuration was used in Ref. 2 to test the Hamiltonian CGMD (without dissipation), and a detailed description of the system is given there. Because the asymptotic regions are described by MD, the reflection coefficient is well defined.

The result of these calculations was that Langevin CGMD essentially eliminated all scattering, as shown in Fig. 2a. If the memory function and the equations of motion could be integrated without error, there would have been no unphysical reflection; the only back-scatter was due to numerical noise.

A recent result of Cai, et al [13], found that wave reflection from a fixed boundary could be essentially eliminated using memory functions. There are two difficulties in implementing their formulation in large-scale simulations. The first is that the memory function couples sites on the boundary that are quite far apart, so that the algorithm is not amenable to parallelization. The second is that the memory is long (the memory function was non-zero for 500 time steps), requiring a large amount of storage. These issues are related. They arise because the memory function must absorb long wavelength, long period waves at the fixed boundary. In CGMD, these are the waves that are allowed to pass through the interface, so they do not need to be absorbed. The result is a memory function that is short-ranged in space and time. The value of the largest matrix element is shown in Fig. $2 \mathrm{~b}$ as a function of time, and it is compared to the memory function for a fixed boundary as in the Cai work. Langevin CGMD shows a substantial improvement.

\section{CONCLUSION}

Coarse-grained molecular dynamics is an implementation of concurrent multiscale modeling linking atomistic regions to continuum regions. In this article we have reviewed some earlier work, with the benefit of our current understanding and experience. Also we described recent results that extend the Hamiltonian CGMD to a dissipative, Langevin formulation. Validation using the elastic wave reflection coefficient and the decay of the memory function has shown a substantial improvement over other techniques.

\section{ACKNOWLEDGMENTS}

We owe many thanks to Jeremy Q. Broughton with whom this work was begun. Support has been provided by DARPA. This work was performed in part under the 

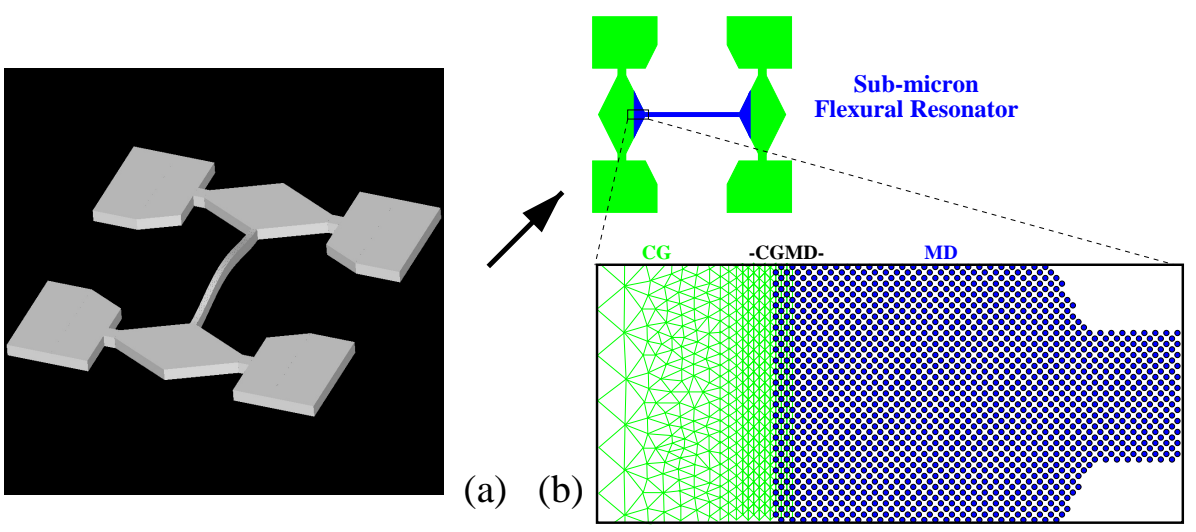

Figure 2: (a) The reflection coefficient for elastic wave scattering off the CG interface (see text) for 4 cases: distributed mass finite element analysis (dist. mass), lumped mass finite element analysis (lump mass), Hamiltonian CGMD (H-CGMD) and Langevin CGMD (LCGMD). Note that H-CGMD has eliminated pathological resonances (peaks) found in finite element analysis, but Langevin CGMD has entirely eliminated scattering (the reflection coefficient is 0 up to numerical noise). (b) A comparison of the memory functions for wave absorption at a hard boundary (cf. Ref. [13]) and at the CG region in CGMD. Note how much shorter the memory is in the CGMD system.

This work was performed under the auspices of the U.S. Department of Energy by the University of California, LLNL under Contract No. W-7405-Eng-48.

\section{REFERENCES}

1. R. E. Rudd and J. Q. Broughton, Phys. Stat. Sol. (b) 217, 251 (2000).

2. R. E. Rudd and J. Q. Broughton, Phys. Rev. B 58, R5893 (1998).

3. R. E. Rudd and J. Q. Broughton, J. Model. and Sim. of Microsys. 1, 26 (1999).

4. C. T.-C. Nguyen, L. P. B. Katehi, and G. M. Rebeiz, Proc. IEEE 86, 1756 (1988).

5. A. N. Cleland and M. L. Roukes, Appl. Phys. Lett. 69, 2653 (1996); See also http://www.cmp.caltech.edu/ roukes/images/mrfm.jpg.

6. D. W. Carr, et al., Appl. Phys. Lett. 75, 920 (1999).

7. J. Q. Broughton, et al., Phys. Rev. B 60, 2391 (1999).

8. E. B. Tadmor, M. Ortiz, and R. Phillips, Philos. Mag. A 73, 1529 (1996).

9. B. L. Holian and R. Ravelo, Phys. Rev. B 51, 11275 (1995).

10. R. Zwanzig, Nonequilibrium Statistical Mechanics, (Oxford Univ. Press, 2001).

11. R. E. Rudd, "Ballistic Transport and Wave Reflection in Coarse-Grained Molecular Dynamics," in preparation, 2001.

12. M. P. Allen and D. J. Tildesley, Computer Simulation of Liquids, (Clarendon Press, Oxford, 1987).

13. W. Cai, M. de Koning, V. V. Bulatov and S. Yip, Phys. Rev. Lett. 85, 3213 (2000). 